\title{
The rules of aging: are they universal? Is the yeast model relevant for gerontology?
}

\author{
Tomasz Bilinski and Renata Zadrag-Tecza ${ }^{\bowtie}$ \\ Department of Biochemistry and Cell Biology, University of Rzeszow, Rzeszów, Poland
}

\begin{abstract}
The success of experimental biology was possible due to the use of model organisms. It is believed that the mechanisms of aging have a universal character and they are conserved in a wide range of organisms. The explanation of these universal mechanisms by tracing survival curves of model organisms clearly suggests that death of individuals is a direct consequence of aging. Furthermore, the use of unicellular organisms like yeast Saccharomyces cerevisiae to explain the aging processes of multicellular organisms runs the risk of oversimplification. Aging is a very complex process and therefore in this paper we present arguments suggesting that some of these fundamental assumptions require a deep rethinking and verification.
\end{abstract}

Key words: aging, age related diseases, longevity, yeast

Received: 28 May, 2014; revised: 15 October, 2014; accepted: 27 October, 2014; available on-line: 11 December, 2014

\section{INTRODUCTION}

The definition of aging encompasses two different, although probably causally connected phenomena. The term senescence describes various adverse effects which decrease efficiency of vital processes and lead to visible structural changes of the organism. Unavoidable death of individuals seems to be a direct consequence of aging. From evolutionary point of view, aging is treated in two ways, as a programmable or not programmable process. Programmable theories treat aging as a process of adaptation, which is a specific mechanism leading to the altruistic death of an individual for the benefit of the population, and thus preventing a too high density of a population (Medawar Theory). However non-programmable theories, among which the most popular is the disposable soma theory of Thomas Kirkwood (Kirkwood, 1977), treat aging as a kind of trade-off between investment in reproduction and maintenance of the somatic cells. In this sense, priority lies in reproduction while aging is just a stochastic accumulation of damage that leads to impairment of functions and consequently to death. This opinion which is probably right, has been recently challenged because a number of arguments were collected suggesting a quasi-programmable character of the proximal causes of death. The hyperfunction hypothesis (Blagosklonny, 2006, 2013), can be considered a part of the Williams theory of antagonistic pleiotropy. Both seem to explain at least some aspects of the aging process. However, the fundamental distinction of soma and germline by Weissmann and disposable soma theory by Kirkwood (1977) present other important aspect of the process.

Study of the aging process requires to designate certain universal criteria that would allow for their analysis regardless of the type of the model organism used. One such criterion which also corresponds to the definition of this process is to increase the mortality rate as a function of time and a decrease in fertility. However, these criteria even though adequate for many organisms, have raised some doubts as to their versatility, especially if we take into consideration the phenomenon of 'negligible senescence'. This term was introduced by Finch in 1990 (Finch et al., 1990) in relation to the specific group of organisms for which the mostly used criteria for aging cannot be used. This group includes among others turtles, rockfish or mole-rats. In these species a typical decrease in fertility or increased mortality with age is not observed. Also, no changes indicating a 'progressive loss of function' with age were observed there. Thus, the question arises about the universality of the aging process in the living world and universality of the mechanisms of aging.

In this paper we concentrate mainly on the phenomenon of senescence which is strongly correlated with the free radical theory of Harman (1956) and the role of the unicellular organisms such as yeast $S$. cerevisiae in explanation of the mechanism of this process.

\section{YEAST AS MODEL ORGANISM IN AN AGING STUDY}

The budding yeast is a very popular model organism. The use of the budding yeast Saccharomyces cerevisiae as a model organism of gerontology was based on two essential assumptions. The first of them is that the existence of the reproduction limit of each single cell is a consequence of the aging process. In other words, it was assumed that unavoidable death of each individual cell is not a side effect of the chosen strategy of reproduction (budding), as was postulated (Mortimer \& Johnston, 1959; Muller et al., 1980), but of the aging process (Egilmez \& Jazwinski, 1989). The second assumption was that as the number of daughters produced by a single cell is rather independent of the conditions of growth

e-mail: retecza@univ.rzeszow.pl

Abbreviations: ARDs, age related diseases; $E E$, external environment; IE, internal environment; IECFD, Internal environment control failure diseases; PDDs, Proliferation and differentiation diseases; PRLS, postreproductive life span; PUFA, polyunsaturated fatty acids; RLS, replicative life span; ROS, reactive oxygen species; SASP, senescence-associated secretory phenotype; SSRs, simple sequence repeats; TLS, total lifespan 
and on the time that reproduction takes, therefore the age and longevity of yeast can be expressed as a number of the daughter cells produced, instead of units of time. In that case the conclusions drawn from the studies based on such unusual units cannot be directly applicable for other organisms. The comparison can be made only if the units used are at least proportional.

The acceptance of these assumptions and also the use of the number of generations as a measure of the age strongly influenced the conclusions of the research on yeast model. Thus, each factor or mutation in the genome resulting in a decrease in the number of daughters produced can be considered as a 'senescence factor', and in turn has the opposite effect to a factor which promotes longevity. However, recent studies (Ganley et al., 2012; Kaeberlein, 2012) encouraged a renewed discussion on the causes of the limited reproductive capacity of yeast and thus questioned applicability of the budding yeast to gerontological studies. These studies indicate a strong relationship between the cell size and its reproductive capacity. The hypertrophy hypothesis was put forward (Bilinski, 2012; Bilinski et al., 2012) trying to explain the origin of the existence of reproduction limit of the budding yeast. The hypothesis was inspired by the previous postulate (Muller et al., 1980) that the existence of this limit is a consequence of an unusual mechanism of cytokinesis known as budding. The second and the most important factor suggesting the alternative mechanism of 'replicative aging' of the budding yeast cell that was not taken into account in early discussions on the possible role of the most spectacular phenotype accompanying this phenomenon was hypertrophy of aged cells. Therefore, the possible role of hypertrophy in replicative aging for a long time was not taken into account. According to this hypothesis, the phenomenon known as 'replicative aging' is not a consequence of aging but a side effect of the choice of budding as a mechanism of cytokinesis. Budding enforces increase of the size of the mother cell during each cell cycle. It leads to hypertrophy (up to tenfold increase of cell volume) which precludes further reproduction. It means that the existence of the reproduction limit is rather not a consequence of accumulation of the 'senescence factor' (Bilinski \& Bartosz, 2006; Bilinski et al., 2012) but has a quasi-programmable character, as was postulated earlier. This hypothesis does not exclude the possibility that the mother cells age, but suggests that cells cease reproduction because of hypertrophy, before the aging process can do it. This hypothesis recently gained a very strong experimental support (Zadrag-Tecza et al., 2009; 2013; Yang et al., 2011; Wright et al., 2013). The existence of an alternative opinion on the origin of replicative aging does not mean that the hypothesis of the 'senescence factor' accumulation (Egilmez \& Jazwinski, 1989) should be abandoned. Potentially both hypotheses can explain various aspects of the phenomenon.

After the hypertrophy hypothesis was put forward, an opinion was presented that hypertrophy could be a consequence of aging (Ganley et al., 2012). The analysis of the mechanism of budding (Hartwell \& Unger, 1977; Woldringh et al., 1993) explains in a satisfactory way the reason why the volume of the cell has to increase during each cell cycle. Hence hypertrophy can be treated as a sufficient condition of the reproductive capacity limit, defined as replicative aging. Therefore the postulate that hypertrophy can be a consequence of aging seems to be redundant, especially that no convincing and testable mechanism causing hypertrophy was postulated. However, a definitive conclusion concerning utility of yeast as a model organism for gerontology requires further arguments. The assumption that the reproductive potential of a single budding yeast cell depends on the rate of the aging process has purely a priori character because slowing down of fundamental cellular processes is visible only at the very end of reproductive phase of yeast life (Mortimer \& Johnston, 1959; Kennedy et al., 1994). The use of yeast as a model organism for gerontology required an important assumption, i.e. that by studying behavior of aged yeast cells one can explain a more general (public) mechanism of aging, and not just a private mechanism specific only for the budding yeast.

Gerontology is in fact a human oriented discipline and the conclusions drawn from experimental studies carried on various model organisms should be applicable to human gerontology, as it was openly declared (Kaeberlein et al., 2007; Fontana et al., 2010; Kaeberlein, 2010). Consequently, similarities of the aging process of humans and yeast should be substantial if it is a valuable model organism for aging studies. In this paper the arguments are presented that the processes which are associate with aging of humans and of the budding yeast cells differ so strongly that it causes a problem for effective use of yeast as a model for gerontology.

\section{UNIVERSALITY OF AGING RULE}

The universality of the aging rule, including yeast is one of the motifs of numerous gerontologist publications (Ganley et al., 2012; Kaeberlein, 2012; Polymenis $\&$ Kennedy, 2012). The only universal feature of living things is that majority of their constituents are far from a thermodynamic equilibrium, and therefore tend to decay. The definitions of aging do not explain the reasons for declining functions or structures. It is generally accepted that aging is a consequence of accumulation of damage. One can find the following sentence: "Recent discoveries suggest that aging is neither driven by accumulation of molecular damage of any cause, nor by random damage of any kind" (Blagosklonny, 2012). However, if we replace the word 'aging' with 'longevity', both opinions stop contradicting. We therefore conclude that these two terms should not be used interchangeably. They simply describe different aspects of the phenomena that accompany our advanced age. Therefore, we will separately describe evolution of aging and evolution of longevity. It is almost generally accepted that aging, understood as defined above, results from accumulation of damage or from the damage itself. Because the term 'damage' is strictly defined we suggest to replace the term 'accumulation of damage' by 'accumulation of ballast or cellular trash', because some 'senescence factors', like misfolded proteins, are formed as a consequence of imperfect functioning of cellular mechanisms, instead of always being a consequence of damage. Besides, such 'senescence factors' as rDNA circles (ERCs) are side products of normal cellular processes and cannot be considered the products of damage. However, if we concentrate on universal sources of sensu stricto damage we could look at the problem from two points of view. We would have to separately take into account the factors responsible for damage and the targets and/or products of damage.

\section{Universality of factors responsible for damage}

There are many factors that cause different types of damage to cell components but only some of them have a universal character and are 'conserved'. The evolution of life forms began under anoxic atmosphere where two 
main destructive factors like heat and irradiation dominated. These factors are 'conserved' and universal. However, the oxygen-evolving photosynthesis of early bluegreen algae resulted in the first pollution of the Earth and a new type of damage (Lane, 2002). We have recently begun to consider oxidative damage caused by reactive oxygen species (ROS) as the main cause of aging. Thus, evolution of oxygen-generating photosynthesis resulted in new threats for living things but at the same time made the photosynthetic process independent of scarce sources of reducing equivalents like sulfide. Thus, even now living things inhabiting anaerobic environments are less endangered to oxidative stress than the aerobic ones. Yeast cells are facultative anaerobes and in some environments have extremely rare contacts with oxygen.

The universality of deleterious mechanisms causing damage becomes even more doubtful when we take into account that human beings evolved an ability to develop specific harmful factors which appear to be a double edged sword. Some types of cells of our immune system can synthesize hypochlorous acid by using primary reactive oxygen species — hydrogen peroxide. This compound emerges in some autoimmune diseases and destroys our tissues. Similarly, superoxide ion generates nitrosative stress by forming different nitrogen containing species during various diseases. Besides that, such signaling but potentially dangerous molecules like leukotrienes and prostaglandins are also absent in yeast.

\section{Universality of targets and products of damage}

In the early Precambrian, mostly proteins and nucleic acids were the targets of damage. The appearance of ROS increased damage to macromolecules. Besides injury resulting from ionizing irradiation and heat, nucleic acids and proteins also began to undergo oxidative damage. Formation of 8-oxyguanosine in DNA and carbonyl groups in proteins are the best known examples (Fraga et al., 1990; Berlett \& Stadtman, 1997). However, the crucial parts of each cell, i.e. the cellular membranes, were much less endangered by oxidative stress. Lowering of the Earth's temperature led to the evolution of numerous oxygen-dependent (hence evolutionary young) fatty acid desaturases, necessary for generation of polyunsaturated fatty acids (PUFA). Poly- in this case means more than one double bond. Low melting temperature of PUFAs enabled life in colder environments and allowed the spread of various life forms to new areas of our globe. These molecules, in contrast to the saturated or monounsaturated fatty acids, are chemically reactive and undergo a process known as lipid peroxidation. This multistep and variable chain of reactions created real problems for living things, starting from structural changes in lipid bilayers to the formation of highly toxic unsaturated aldehydes (Esterbauer et al., 1991). Simultaneous oxidation of proteins and lipids resulted in the formation of cross-linked products like age pigments (lipofuscins). These products do not undergo enzymatic degradation and thus accumulate within the cells. Lipofuscins are toxic and phototoxic. They accumulate in all postmitotic cells and their accumulation in the skin is one of the best known visible symptoms of aging (Sohal \& Brunk, 1989). Their accumulation in the intestine is one of the most important biomarkers of aging in Caenorbabditis (Klass, 1977; Garigan et al., 2002). Additionally unsaturated aldehydes, known as products of lipid peroxidation, could generate enormous amounts of glutathione conjugates which could be accumulated within the cell. Some aldehydes form insoluble adducts with proteins which also accumulate. Thus the appearance of oxygen and PUFA not only strongly increased the level of damage, but also broadened the spectrum of vulnerable targets. What is however most important, even hydrophobic parts of the cell (membranes and lipid droplets) also became endangered. Even though considered as a preeminent model organism for gerontology (Ganley et al., 2012; Kaeberlein, 2012; Polymenis \& Kennedy, 2012) yeast are unable to synthesize PUFA and therefore their lipids are not subjected to this dangerous threat. Thus, accumulation of oxidative damage in yeast and human cells is incomparable. Hence, even universality of the mechanisms of oxidative damage is easy to question. Increase in chemical complexity of living things created new dangers, but also increased the number of vulnerable targets and as a consequence formed new toxic products of their damage.

\section{Universality of protective mechanisms}

Oxidative processes are considered the main cause of damage associated with aging. Investment in protective and repair mechanisms is considered a very important strategy of counteracting aging and increasing longevity (Calabrese et al., 2012). Therefore the involvement of various antioxidants in preventing oxidative and photo damage can be also considered a part of the aging process. All aerobic organisms share similar basic antioxidant mechanisms, although despite these similarities Saccharomyces cerevisiae differs from plants and animals in some of them. Lack of damage connected to the lipid peroxidation process in Saccharomyces cerevisiae is accompanied by the absence of two antioxidants like tocopherol and ascorbate, important for human beings. Similarly, availability of various types of carotenoids in humans is an important factor preventing damage to the eye and skin and influencing the health status (Donaldson, 2011; Ozawa et al., 2012). The role of carotenoids in the budding yeast protection is negligible.

\section{Increased complexity of cells changed aging rules}

The consequences of evolving complexity of living things are not restricted to the appearance of new types of targets and products. Formation of eukaryotic cells resulted in the appearance of new problems that we associate with aging. For example, increasing the amount of genetic information led to linearity of DNA and dividing it into packages (chromosomes). Linearity enforced formation of telomeres. Shortening of telomeres in some somatic cells is considered one of the aspects of human aging (Aubert \& Lansdorp, 2008). In contrast to human somatic cells, the enzyme telomerase is always active in yeast (Cohn \& Blackburn, 1995) however, it does not allow for an unlimited number of reproductive cycles. On the other hand, dividing genetic information into chromosomes necessitated their precise transfer to descendant cells, which gave rise to a new source of errors (chromosomal aberrations). Formation of chromatin protected DNA against damage and formation of nuclear envelope further improved this protection. However, some organisms like yeast evolved a simpler type of karyokinesis known as closed mitosis. It differs from open mitosis, known in animals and plants. In closed mitosis, nuclear envelope never disintegrates and as a consequence the side products of homologous recombination of DNA - rDNA circles (ERCs) accumulate. It is worthwhile to emphasize that some yeast researchers considered these episomes the main causative factors of replicative aging in yeast (Sinclair \& Guarente, 
1997). Hence, this accumulation is a 'private' feature of this group of organisms and it is difficult to postulate its direct relation to human aging.

Evolution of the endoplasmic reticulum in eukaryotes, which is an oxidizing compartment facilitating the formation of disulfide bonds in proteins, resulted in increased oxidation of resident proteins of this compartment. It is characteristic that proteins that underwent oxidative damage and contain carbonyl groups are considered as another causative factor of replicative aging of the budding yeast. The fact that most of the fluorescent signal coming from carbonylated proteins is located within the mother cell is interpreted as a consequence of the existence of specific mechanisms retaining damage within the mother cell, which 'rejuvenates' the daughter cells. Because resident proteins of ER are the main targets of oxidative damage, we can interpret these effects as a consequence of the presence of ER (its perinuclear part) within the mother cell for most of the cell cycle.

The existence of the vacuole in yeast (absent in human cells) also should be considered. During most of the cell cycle the vacuole stays within the mother cell, and thus most of the conjugated glutathione residing within this vacuole cannot be equally partitioned between both products of cytokinesis (Banta et al., 1988; Klionsky et al., 1990; DeMesquita et al., 1997). Vacuole is also the main iron storage compartment in yeast $(\mathrm{Li}$ et al., 2001). Vacuole becomes a 'junkyard' for yeast cells. On the contrary the presence of homologous organelles - lysosomes in humans, create serious problems like SASP during cellular senescence of human somatic cells (Coppe et al., 2008; van Deursen, 2014) which is not encountered in yeast.

Thus, in budding yeast, specific elements of their biology created new problems, resulting in the accumulation of group-specific trash. All these facts clearly disprove the universality of the aging process even between fungi and humans. Various aspects of the aging process evidently developed in parallel as the cells themselves evolved. The above does not apply to the aging of prokaryotic organisms.

\section{Unusual life strategies can result in rare types of senescence}

In Paramecium the strategy of being a giant among unicellular organisms, which assures predatory way of feeding, resulted in the necessity of evolving a macronucleus containing around one thousand copies of chromosomes (Kimura et al., 2004). Although this "kiloploidy" of the vegetative nucleus assures a high rate of mitotic reproduction, at the same time it results in degradation of this organelle after a number of mitotic cycles. Unusual quantitative increase of ploidy caused mechanistic problems in precise manipulation of such a high number of chromosomes during karyokinesis, which rarely takes place in the case of haploids or oligoploids.

\section{LONGEVITY}

Evolution of the structure of genomes from compact, like in bacteria and budding yeast (except introns and SSRs) to those dominated by noncoding DNA of vertebrates strongly influenced various aspects of life including our longevity. Alternative splicing is another important difference between yeast and humans. Longevity of various animals and human beings strongly depends on the morbidity of various diseases. It is known that some ARDs are functionally connected with telomere shorten- ing such as idiopathic pulmonary fibrosis, bone marrow failure, and cryptogenic liver cirrhosis (Armanios, 2013). In the last couple of years a completely new problem has emerged. It is known that in a number of ARDs the micro RNA world is involved. The most recent data suggest that hundreds of diseases including diabetes are in some way connected with formation of these small molecules (Tufekci et al., 2014). Breast cancer metastasis is also functionally associated with micro-RNAs (GuerraAssuncao \& Enright, 2010). It is worth noting that yeast Saccharomyces cerevisiae cells are considered as negative control in experiments concerning miRNAs. The budding yeast genome presumably does not contain noncoding RNAs. The fission yeast Schizosaccharomyces pombe has smaller genome (4970 ORFs) than Saccharomyces cerevisiae (5600 ORFs) but shows many more similarities to mammalian cells than the latter. Genome of $S$. pombe contains 450 noncoding RNAs and is able to process microRNA. It has many more introns - about 5000 when compared to 250 in S. cerevisiae. Hence even in the group of unicellular and apparently similar organisms occupying the same niches, no uniformity of some basic life strategies can be found. Surprisingly the budding yeast, which are biochemically, structurally and functionally distant organism, appeared to be chosen a 'preeminent' model of human aging research. Taking into account that these two mechanisms, i.e. telomere shortening and involvement of miRNAs does not concern yeast, the mechanisms influencing longevity of both species seem to be incomparable.

An additional problem in this case is also a way of expressing longevity by presenting only the number of generations. However, there is a way that requires only a small change of protocols used during yeast replicative aging studies. It allows for determining not only the value of RLS (the number of daughter cells produced), but also to measure yeast longevity expressed in units of time (Minois et al., 2005). This simple modification allowed for determining the value of the post reproductive life span (PRLS) of yeast cells and simultaneously a total life span (TLS) of yeast (Zadrag et al., 2008). Using this procedure, longevity of various mutants can be presented in units comparable to other organisms. The discovery that the life span of yeast cells consists of two phases: reproductive and post-reproductive significantly changed the viewing of this phenomenon. One of the fundamental methodical bases of yeast aging paradigm was the assumption that the cell that ceased reproduction is dead. According to the new protocol yeast longevity can be expressed in two ways. The first one uses the number of daughters produced (RLS) as a measure of longevity. The new one presents longevity in units of time (TLS), like in the case of other organisms. It is important especially if we use the term 'longevity', because this term is directly associated with 'time' not with 'reproduction potential'.

Deletion of numerous genes results in increasing the value of RLS (Kaeberlein et al., 2005a; Smith et al., 2008). These deletion mutants were named 'longevity' mutants. Some of these genes have homologs in other organisms suggesting the possibility of formulating some general conclusions regarding the mechanism of their action like the TOR pathway that occurs in all eukaryotes and has substantially similar functions. However, despite the similarity of the mechanism, the consequences of a deletion of a particular gene may be dependent on the type of organism and its life strategy. Deletion of the TOR1 gene in yeast causes an increase in the number of daughters produced (RLS) (Kaeberlein et al., 2005b), but there 
is no published information about the influence of this mutation on the time of life (TLS). Our preliminary data suggest that the time of life of the mutants does not differ from that of the standard strain (unpublished data). In some cases, the negative correlation of PRLS and RLS (Zadrag-Tecza et al., 2013) causes that the life span of various yeast mutant cells expressed in units of time does not differ substantially from the standard strains. The strains differing up to five times in the RLS value have almost identical TLS. Therefore, naming yeast mutants with higher than standard value of RLS the 'longevity' mutants requires their retesting with the use of improved method of tracing their survival curves.

\section{Multicellularity created a completely new problem}

If we look at multicellular organisms (Metazoans and vascular plants) from the point of view of gerontology it becomes obvious that they differ from unicellular ones in one fundamental aspect. Death of animals, which are multicellular organisms, results mainly from diseases strictly connected with multicellularity. To explain this we will concentrate mainly on human beings.

What are the main causes of death in humans? The answer is not simple. Survival curves of the citizens of developed countries are so strongly influenced by high life standards, including prophylactics and medical care. Therefore, taking into account purely biological factors we should concentrate on survival curves of primitive tribes or data collected centuries ago. Although lifespans were much shorter than now, the influence of the aging process was surprisingly high, because such effects of the aging process as deafness, poor sight and even defects in other senses played important roles in survival. This resulted in deaths connected with accidents, predation and starvation.

The second important factor, recently rather less important, are infectious diseases which threatened human populations partly irrespective of age of individuals. Mortality curves of contemporary populations are shaped mostly by age related diseases except people who survived until a really advanced age. Many of ARDs played marginal roles in primitive tribes, whose mean age was very low. Therefore, taking into account biological potential of our species, human beings mainly die because of various types of diseases, including ARDs.

We can ascribe diseases to two main functional groups, which jointly account for the majority of human deaths. This classification, although it does not exclude common etiology of particular diseases, seems to be useful from a mechanistic point of view. It emphasizes the differences between uni- and multicellular organisms.

\section{INTERNAL ENVIRONMENT CONTROL FAILURE DISEASES (IECFD)}

We have introduced the term internal environment (IE) to emphasize the main functional difference between unicellular and multicellular organisms. This term describes all mechanisms assuring homeostasis of our body. We will try to visualize these fundamental dissimilarities by analyzing the role of the mechanisms controlling the glucose level. Mutations in insulin-like pathways are thought to strongly influence longevity of animals. At the same time, some researchers voice opinions represented by the following sentence: "Although yeast do not have an insulin-signaling pathway, they appear to have precursors of such a metabolic control pathway that function in the glucose/nutrient-signaling cascade and are homologous to the serine/threonine kinase Akt/PKB of insulin-signaling pathways in C. elegans and mammals" (Barbieri et al., 2003). This statement seems to convince readers that the elements of this crucial regulatory pathway preexisted in unicellular organisms. We agree that the sequences of proteins engaged in new pathways should preexist in simpler organisms, but the assumptions that even their functions should preexist seems irrational. The most important difference between unicellular organisms and multicellular ones is that unicellular organisms cannot control their environment. Unicellular organisms can mainly deteriorate the EE (external environment) by excreting various toxic products of metabolism. Unicellular organisms such as yeast have to monitor changes in the environment in order to rapidly adapt to modified availability of nutrients. Yeast preferentially take up fermentable sources of carbon and energy. When the population grows exponentially, the concentration of glucose rapidly drops, which could result in an energy starvation that is dangerous for reproducing cells. This is why the concentration of glucose in the medium has to be precisely monitored by yeast cells to assure enough time to switch to oxidative mode of ATP formation. Yeast metabolism is preferentially fermentative.

In contrast, multicellular organisms can properly function only if they precisely control their internal environment. This is possible because of separation of IE from $\mathrm{EE}$ and limited volume of the IE. Precise control of the nutrient concentration in the body fluids assures homeostasis. In Metazoans the concentration of glucose should be not only monitored, like in yeast, but also controlled. Somatic cells are supplied with glucose, the concentration of which is constant. Only few and very specialized cells both monitor its level and immediately control it by excreting into the IE the appropriate signaling molecules, like insulin or glucagon. Efficiency of this control is possible due to the existence of specific organs like liver or a fat body in insects, which immediately respond to signals, polymerizing glucose or depolymerizing polysaccharides. Hence, glucose control mechanisms are strictly connected to multicellularity, differentiation and organogenesis. They assure separation of monitoring/control elements like specialized pancreatic $\alpha$ and $\beta$ cells are separated in the controlling organ from an executing organ - liver or fat body. The rest of the cells profit from this solution. Although glucose level control seems to be universal in Metazoans, in Drosophila the same system simultaneously controls the level of lipids. Thus even similar control mechanisms are not necessarily universal. It is important to remember that in Drosophila the miR-14 regulates insulin production and metabolism (Varghese et al., 2010). Thus, suggesting that common mechanisms controlling glucose level exist in yeast (that are unable to process micro RNAs) and insects or mammals are not based on solid grounds.

The role of IE in Metazoans is not limited to assuring proper glucose and other nutrient concentration. It is also responsible for the removal of toxic products of metabolism, facilitating real homeostasis. The IE usually also assures oxygen supply (except insects).

The very important function of the IE is the protection of the body against pathogens.

If we consider the major reasons of human death from this perspective, it becomes evident that most of the diseases threatening human populations are connected with problems of improper functioning of the IE. It is worth noting that homeostasis, crucial for the functioning of all cells in the body can be achieved by the 
circulatory system. Thus, such diseases connected to the IE like cardiovascular, renal and related to the protection against pathogens dominate as the causes of death. These factors do not exist in yeast cells. Human longevity also depends to some extent on the presence of PUFAs. Arachidonate is the source of prostaglandins, tromboxanes and leukotrienes, i.e. locally acting hormone like molecules. These molecules are not synthesized by yeast.

\section{PROLIFERATION AND DIFFERENTIATION DISEASES (PDDS)}

Diseases which result from erroneous differentiation and loss of control over cell proliferation are also connected to multicellularity. Various types of malignancies strongly influence mortality curves of human beings. PDDs are also the consequences of multicellularity because the mechanisms preventing reproduction of cells in unicellular organisms have no biological sense. In contrast, strict limitation of cell proliferation is a must in Metazoans. The morbidity from malignancies is to some extent also connected with proper functioning of the immune system eliminating most of malignant cells, i.e. with the internal environment. Although etiology of these diseases is not uniform, they influence our survival curves in a similar way, but mechanically distant from IECFD.

This survey of new threats casts doubt on the notion that the factors influencing longevity of yeast and human beings are universal.

\section{Reproductive potential of cells and longevity of human beings}

The assumption that analysis of the factors influencing yeast longevity (expressed as reproductive potential of cells) could explain the mechanisms of longevity of human beings, made this unicellular organism a model organism for gerontology. Survival curves of humans are shaped by the consequences of diseases strictly connected to multicellularity, not directly with the reproductive potential of our cells. Even the relations between cellular senescence in vitro and in vivo are not clear. We rarely exhaust reproductive potential of our somatic cells that are able to reproduce. When regarding the role of the cellular senescence in longevity of human organism as a whole, the relation of reproduction limit of the cell to organism's longevity is not straight forward. Recently the consequences of cellular senescence for the organism are considered not only to be negative, because it also contributes to cancer prevention and tissue regeneration (Munoz-Espin \& Serrano, 2014). It is now known that the number of senescent (not reproducing) cells increases with age. The growing number of these cells negatively influences their environment by secretion of many factors, like cytokines and chemokines (Campisi, 2001), which could trigger a variety of cellular responses and in this way contribute to various diseases and therefore to longevity. Consequently, the post reproductive period of their life becomes dangerous to the organism as a whole, not the fact that they ceased reproduction.

One of the basic elements of yeast gerontology paradigm is that the cells that ceased reproduction are considered to be dead. It appeared evidently not to be true (Minois et al., 2005). The existence of their post reproductive period of life, so important in the case of human senescent somatic cells, is simply ignored in the case of yeast. Obviously these cells which ceased reproduction are so rare in the growing yeast population that they cannot influence its fate. Therefore, even poor analogy between the existence of reproduction limit of yeast and human cells becomes questionable when their role in the organism's longevity is concerned.

Thus, tracing mortality curves of unicellular organisms, known to have limited reproductive capacity, cannot explain factors influencing longevity of animals or human beings, although they have almost identical shapes (Sinclair et al., 1998).

\section{CONCLUSION}

The survey of differences between various threats associated with the aging process of the budding yeast Saccharomyces cerevisiae and human beings shows that their basic life processes strongly differ. Therefore the use of yeast in gerontological studies, aimed at explaining the mechanisms of human aging, does not seem to be well founded. The analysis of methodology of yeast gerontological studies shows that the basic tool used - tracing 'survival' curves cannot reveal fundamental mechanisms of aging. Finding an ideal model organism to explain the mechanisms of human aging seems impossible since even the use of mouse model in studies on cellular senescence in humans has been recently questioned (van Deursen, 2014), though evolutionary distance between these two mammals is much shorter than in the case of yeast.

\section{Acknowledgements}

This work was supported by Grant no. DEC2013/09/B/NZ3/01352 from the Polish National Science Centre.

\section{REFERENCES}

Armanios M (2013) Telomeres and age-related disease: how telomere biology informs clinical paradigms. J Clin Invest 123: 996-1002.

Aubert G, Lansdorp PM (2008) Telomeres and aging. Physiol Rev 88: 557-579.

Banta LM, Robinson JS, Klionsky DJ, Emr SD (1988) Organelle assembly in yeast - characterization of yeast mutants defective in vacuolar biogenesis and protein sorting. J Cell Biol 107: 1369-1383.

Barbieri M, Bonafe M, Franceschi C, Paolisso G (2003) Insulin/IGFI-signaling pathway: an evolutionarily conserved mechanism of longevity from yeast to humans. AM J Physiol-Endoc M 285: E1064E1071.

Berlett BS, Stadtman ER (1997) Protein oxidation in aging, disease, and oxidative stress. I Biol Chem 272: 20313-20316.

Bilinski T (2012) Hypertrophy, replicative ageing and the ageing process. FEMS Yeast Res 12: 739-40.

Bilinski T, Bartosz G (2006) Hypothesis: cell volume limits cell divisions. Acta Biochim Pol 53: 833-835.

Bilinski T, Zadrag-Tecza R, Bartosz G (2012) Hypertrophy hypothesis as an alternative explanation of the phenomenon of replicative aging of yeast. FEMS Yeast Res 12: 97-101.

Blagosklonny MV (2006) Aging and immortality — Quasi-programmed senescence and its pharmacologic inhibition. Cell Cycle 5: 2087-2102.

Blagosklonny MV (2012) Answering the ultimate question "What is the Proximal Cause of Aging?". Aging-Us 4: 861-877.

Blagosklonny MV (2013) Aging is not programmed Genetic pseudoprogram is a shadow of developmental growth. Cell Cycle 12: 37363742 .

Calabrese V, Cornelius C, Dinkova-Kostova AT, Iavicoli I, Di Paola R, Koverech A, Cuzzocrea S, Rizzarelli E, Calabrese EJ (2012) Cellular stress responses, hormetic phytochemicals and vitagenes in aging and longevity. BBA-Mol Basis Dis 1822: 753-783.

Campisi J (2001) From cells to organisms: can we learn about aging from cells in culture? Exp Gerontol 36: 607-618.

Cohn M, Blackburn EH (1995) Telomerase in yeast. Science 269: 396400.

Coppe J-P, Patil CK, Rodier F, Sun Y, Munoz DP, Goldstein J, Nelson PS, Desprez P-Y, Campisi J (2008) Senescence-Associated Secretory Phenotypes Reveal Cell-Nonautonomous Functions of Oncogenic RAS and the p53 Tumor Suppressor. Plos Biology 6: 2853-2868. 
DeMesquita DSG, Shaw J, Grimbergen JA, Buys MA, Dewi L, Woldringh CL (1997) Vacuole segregation in the Saccharomyces cerevisiae vac2-1 mutant: Structural and biochemical quantification of the segregation defect and formation of new vacuoles. Yeast 13: 999-1008.

Donaldson MS (2011) A Carotenoid Health Index Based on Plasma Carotenoids and Health Outcomes. Nutrients 3: 1003-1022.

Egilmez NK, Jazwinski SM (1989) Evidence for the involvement of a cytoplasmic factor in the aging of the yeast Saccharomyces cerevisiae. I Bacteriol 171: 37-42.

Esterbauer H, Schaur RJ, Zollner H (1991) Chemistry and biochemistry of 4-hydroxynonenal, malonaldehyde and related aldehydes. Free Radical Bio Med 11: 81-128.

Finch CE, Pike MC, Witten M (1990) Slow mortality-rate accelerations during aging in some animals approximate that of humans. Science 249: 902-905.

Fontana L, Partridge L, Longo VD (2010) Extending Healthy Life Span-From Yeast to Humans. Science 328: 321-326.

Fraga CG, Shigenaga MK, Park JW, Degan P, Ames BN (1990) Oxidative damage to DNA during aging - 8-hydroxy-2'-deoxyguanosine in rat organ DNA and urine. Proc Natl Acad Sci USA 87: 45334537.

Ganley ARD, Breitenbach M, Kennedy BK, Kobayashi T (2012) Yeast hypertrophy: cause or consequence of aging? Reply to Bilinski et al. FEMS Yeast Res 12: 267-268.

Garigan D, Hsu AL, Fraser AG, Kamath RS, Ahringer J, Kenyon C (2002) Genetic analysis of tissue aging in Caenorhabditis elegans: A role for heat-shock factor and bacterial proliferation. Genetics 161: 1101-1112.

Guerra-Assuncao JA, Enright AJ (2010) MapMi: automated mapping of microRNA loci. BMC Bioinformatics 11.

Harman D (1956) Aging — a theory based on free-radical and radiation-chemistry. Journals of Gerontology 11: 298-300.

Hartwell LH, Unger MW (1977) Unequal division in Saccharomyces cerevisiae and its implications for control of cell division. J Cell Biol 75: $422-435$.

Kaeberlein M (2010) Lessons on longevity from budding yeast. Nature 464: 513-519.

Kaeberlein M (2012) Hypertrophy and senescence factors in yeast aging. A reply to Bilinski et al. FEMS Yeast Res 12: 269-270.

Kaeberlein M, Burtner CR, Kennedy BK (2007) Recent developments in yeast aging. PLOS Genet 3: 655-660.

Kaeberlein M, Kirkland KT, Fields S, Kennedy BK (2005a) Genes determining yeast replicative life span in a long-lived genetic background. Mech Ageing Dev 126: 491-504.

Kaeberlein M, Powers RW, Steffen KK, Westman EA, Hu D, Dang N, Kerr EO, Kirkland KT, Fields S, Kennedy BK (2005b) Regulation of yeast replicative life span by TOR and Sch9 in response to nutrients. Science 310: 1193-1196.

Kennedy BK, Austriaco NR, Guarente L (1994) Daughter cells of Saccharomyces cerevisiae from old mothers display a reduced life-span. J Cell Biol 127: 1985-1993.

Kimura N, Mikami K, Endoh H (2004) Delayed degradation of parental macronuclear DNA in programmed nuclear death of Paramecium caudatum. Genesis 40: 15-21.

Kirkwood TBL (1977) Evolution of aging. Nature 270: 301-304.

Klass MR (1977) Aging in nematode Caenorhabditis elegans - major biological and environmental factors influencing life-span. Mech Ageing Dev 6: 413-429.

Klionsky DJ, Herman PK, Emr SD (1990) The fungal vacuole composition, function, and biogenesis. Microbiol Rev 54: 266-292.
Lane N (2002) Oxygen: The molecule that Made the World. Oxford University Press.

Li LT, Chen OS, Ward DM, Kaplan J (2001) CCC1 is a transporter that mediates vacuolar iron storage in yeast. J Biol Chem 276: 2951529519.

Minois N, Frajnt M, Wilson C, Vaupel JW (2005) Advances in measuring lifespan in the yeast Saccharomyces cerevisiae. Proc Natl Acad Sci USA 102: 402-406.

Mortimer RK, Johnston JR (1959) Life span of individual yeast cells. Nature 183: 1751-1752.

Muller I, Zimmermann M, Becker D, Flomer M (1980) Calendar life span versus budding life span of Saccharomyces cerevisiae. Mech Ageing Dev 12: 47-52.

Munoz-Espin D, Serrano M (2014) Cellular senescence: from physiology to pathology. Nat Rev Mol Cell Bio 15: 482-496.

Ozawa Y, Sasaki M, Takahashi N, Kamoshita M, Miyake S, Tsubota K (2012) Neuroprotective effects of lutein in the retina. Curr Pharm Design 18: 51-56.

Polymenis M, Kennedy BK (2012) Chronological and replicative lifespan in yeast: Do they meet in the middle? Cell Cycle 11: 3531-3532.

Sinclair D, Mills K, Guarente L (1998) Aging in Saccharomyces cerevisiae. Annu Rev Microbiol 52: 533-560.

Sinclair DA, Guarente L (1997) Extrachromosomal rDNA circles - A cause of aging in yeast. Cell 91: 1033-1042.

Smith ED, Tsuchiya M, Fox LA, Dang N, Hu D, Kerr EO, Johnston ED, Tchao BN, Pak DN, Welton KL, Promislow DEL, Thomas JH, Kaeberlein M, Kennedy BK (2008) Quantitative evidence for conserved longevity pathways between divergent eukaryotic species. Genome Res 18: $564-570$.

Sohal RS, Brunk UT (1989) Lipofuscin as an indicator of oxidative stress and aging. In Lipofuscin and Ceroid Pigments, Porta EA ed, pp 17-29. City.

Tufekci KU, Oner MG, Meuwissen RLJ, Genc S (2014) The Role of MicroRNAs in Human Diseases. Methods in Molecular Biology (Clifton NJ) 1107: 33-50.

van Deursen JM (2014) The role of senescent cells in ageing. Nature 509: 439-446.

Varghese J, Lim SF, Cohen SM (2010) Drosophila miR-14 regulates insulin production and metabolism through its target, sugarbabe. Gene Dev 24: 2748-2753.

Woldringh CL, Huls PG, Vischer NOE (1993) Volume growth of daughter and parent cells during the cell-cycle of Saccharomyces cerevisiae a/alpha as determined by image cytometry. J Bacteriol 175: 3174-3181.

Wright J, Dungrawala H, Bright RK, Schneider BL (2013) A growing role for hypertrophy in senescence. FEMS Yeast Res 13: 2-6.

Yang J, Dungrawala H, Hua H, Manukyan A, Abraham L, Lane W, Mead H, Wright J, Schneider BL (2011) Cell size and growth rate are major determinants of replicative lifespan. Cell Cycle 10: 144-155.

Zadrag-Tecza R, Kwolek-Mirek M, Bartosz G, Bilinski T (2009) Cell volume as a factor limiting the replicative lifespan of the yeast Saccharomyces cerevisiae. Biogerontology 10: 481-488.

Zadrag-Tecza R, Molon M, Mamczur J, Bilinski T (2013) Dependence of the yeast Saccharomyces cerevisiae post-reproductive lifespan on the reproductive potential. Acta Biochim Pol 60: 111-115.

Zadrag R, Bartosz G, Bilinski T (2008) Is the yeast a relevant model for aging of multicellular organisms? An insight from the total lifespan of Saccharomyces cerevisiae. Curr Aging Sci 1: 159-65. 